\title{
Base excitation in one-dimensional soil dynamics
}

\author{
E. Benjamin Wylie \\ Department of Civil Engineering, University of Michigan, Ann Arbor, MI 48109-2125, USA
}

\author{
and Chien-Tai Wu \\ University of Michigan, Ann Arbor, MI 48109-2125, USA
}

\begin{abstract}
The prescribed motion to be used as base excitation for shear wave propagation studies in soils is not well defined in the literature. This paper utilizes two methods to investigate the concept of utilizing one-half the rock surface velocity as the incident velocity at a transmitting boundary at the soil base. A range of material properties, thicknesses, and frequencies are used to explore the variation of the ratio: incident velocity to rock surface velocity. Although the ratio varies over a broad range recommendations are made to assist the analyst in selecting a conservative ratio suitable for engineering studies.
\end{abstract}

Key Words: analysis, one-dimensional, shear wave, soil dynamics, transmitting boundary

\section{INTRODUCTION}

In the study of shear wave propagation in soils, the soil base motion is seldom available. Most generally, only recorded rock surface seismographs are available, either from measurements in the vicinity of the site or measurements that are transposed from other locations. These recorded surface motions are often imposed as the actual total velocity at soil base. This method produces unreliable results that are most often in excess of the physical response. An alternative procedure involves inverting the rock surface velocity to get the incident velocity at a substrata interface. Preferably the base material below the interface is homogeneous. The method uses this computed incident velocity as the prescribed motion at the same level beneath the soil to compute the response of soil mass.

An alternative method uses one-half the rock surface velocity as the incident velocity at the soil base ${ }^{3}$. This method, more convenient but not so precise, provides a similar soil response. However, to create the same amplitude, the method requires multiplication by a constant different from one-half in some instances. It is the objective of this study to find this constant and to identify its range of applicability. The potential simplification in soil motion analysis provides the motivation.

Two methods are used to find the constant: namely a first pulse method, and a frequency domain method. The former one considers the constant to be a function of material properties only. The latter one considers the constant to be a function of both material properties and wave frequency. The latter method is more complete and therefore provides a more reliable estimate of the constant. Rock without dissipation is first assumed to compute the constant; later the limitations imposed by this assumption are investigated.

\section{PHYSICAL DOMAIN DESCRIPTION}

The domain under consideration for both methods is shown in Figure 1. The material in each layer is locally

Accepted June 1984. Discussion closes March 1985.

0261-7277/85/010024-07\$2.00

(C) 1985 CML Publications homogeneous. Two types of stratification are investigated. The first type, called TP1, is the one in which the material property becomes progressively weaker when measured from the base level to the surface. The second type, called TP2, contains the opposite type of stratification, namely weak to strong from base to surface. Typically the material is rock with shear moduli, $G$, in the range $9 \times 10^{8}$ to $7 \times 10^{9} \mathrm{psf}$, maximum shear stress, $\tau_{\max }$, in the range $2 \times 10^{6}$ to $7 \times 10^{6} \mathrm{psf}$, and specific gravity, $S$ in the range 2.5 to $3.5^{5}$. The number of layers above the base level is represented by $n$, which can be any number other than unity. It is desired to compute the ratio, $r$, between the base incident velocity and the total surface velocity.

\section{INITIAL PULSE METHOD}

An approximate, but illustrative, method is presented first. This method, which considers only the response due to the initial pulse, provides a simple relationship between base incident velocity and the total surface velocity.

In Figure 2, initially there is an incident wave which is assumed to have originated at a depth several thousand feet below the free surface. From the method of characteristics $^{6}$, the following equation is valid for motion at any arbitrary interface $f_{m}$,

$$
C_{m}^{-}: \tau_{T_{m}}=\tau_{I_{m}}-\rho_{m} v_{s_{m}}\left(V_{l_{m}}-V_{T_{m}}\right)
$$

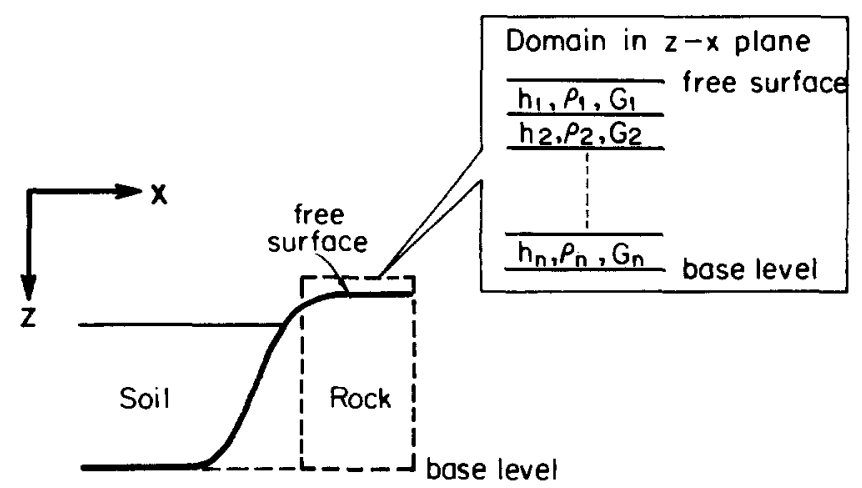

Figure 1. Physical representation of domain in the $z-x$ plane 

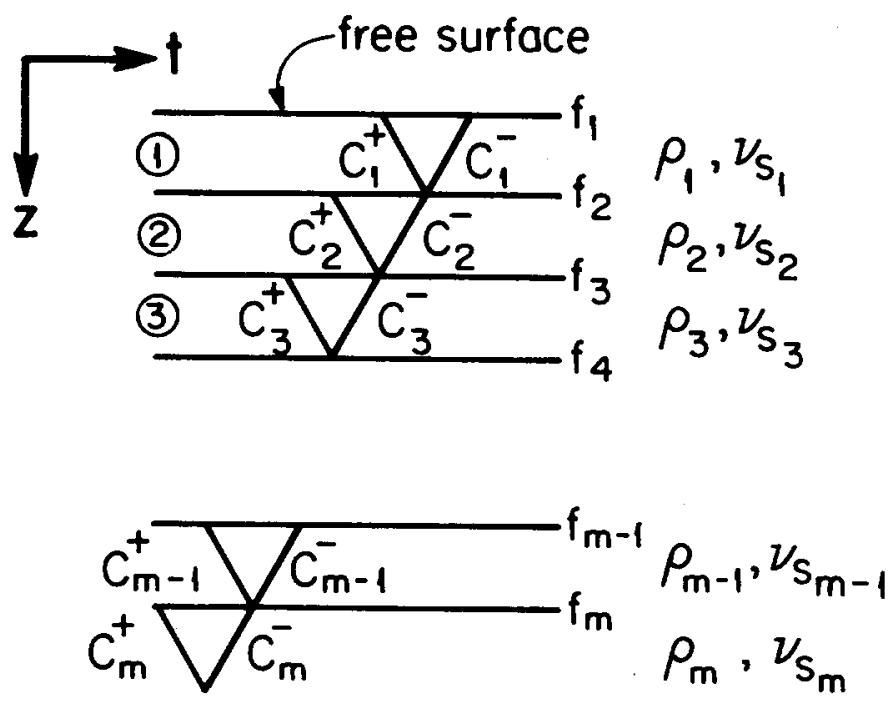

Figure 2. Characteristic lines in the $z-t$ plane

$C^{-}$designates a negatively sloped characteristic line, $\tau_{T}=$ total shear stress, $\tau_{I}=$ incident shear stress, $V_{T}=$ total velocity, $V_{l}=$ incident velocity, $\rho=$ mass density, $v_{s}=$ shear wave propagation, and the subscript $m$ designates any arbitrary number for both the layer and the interface directly above.

Since there is no previous motion in the rock, along any $C_{m-1}^{+}$the relation between total shear stress, $\tau_{T_{m}}$ and total velocity, $V_{T_{m}}$ is

$$
C_{m-1}^{+}: \tau_{T_{m}}=\rho_{m-1} v_{s_{m-1}} V_{T_{m}}
$$

and along any $C_{m}^{+}$characteristic line, the relation between incident shear stress, $\tau_{I_{m}}$, and incident velocity, $V_{I_{m}}$, is

$$
C_{m}^{+}: \tau_{I_{m}}=\rho_{m} v_{s_{m}} V_{I_{m}}
$$

The combination of Eqs. (1), (2), and (3) yields

$$
V_{T_{m}}=\frac{2}{1+\frac{\rho_{m-1} v_{s_{m-1}}}{\rho_{m} v_{s_{m}}}} V_{I_{m}}
$$

In any $(m-1)$ th layer, the material property is locally homogeneous; thus,

$$
V_{I_{m-1}}=V_{T_{m}}
$$

When the computation proceeds from a bottom layer $n$ upwards to layer 1, repeated application of Eqs. (4) and (5), with index notation, $m$, changing from $n$ to 1 , and $\rho_{0} v_{s_{0}}=0$, yields:

$$
V_{T_{1}}=2\left(\prod_{m=2}^{n} \frac{2}{1+\frac{\rho_{m-1} v_{s_{m-1}}}{\rho_{m} v_{s_{m}}}}\right) V_{l_{n}}
$$

in which $V_{T_{1}}=$ total surface velocity, $V_{I_{n}}=$ base incident velocity, and the notation $\prod_{m=2}^{n}$ refers to the multiplication operator. From Eq. (6), the ratio, $r$, is

$$
r=\frac{V_{l_{n}}}{V_{T_{1}}}=\frac{1}{2}\left(\prod_{m=2}^{n} \frac{1+\frac{\rho_{m-1} v_{s_{m-1}}}{\rho_{m} v_{s_{m}}}}{2}\right)
$$

It is convenient to define $A_{m-1}=\left(\rho_{m-1} v_{s_{m-1}} / \rho_{m} v_{s_{m}}\right)$. The wave propagation velocity is $v_{s}=(\mathrm{G} / \rho)^{1 / 2}$ for a material without energy dissipation; therefore,

$$
A_{m-1}=\left(\frac{\rho_{m-1} \mathrm{G}_{m-1}}{\rho_{m} \mathrm{G}_{m}}\right)^{1 / 2}
$$

Values of the velocity ratio are easily computed with Eq. (7). Limiting values of the ratio are of particular interest. Applying a Taylor expansion and Cauchy inequality equation, one can prove, Appendix, that $r$ has a limit no matter how many layers there are in the rock, and the limit occurs at the condition when $A_{1}=A_{2}=\ldots=A_{n-1}$. The result in Appendix is $r_{\min }=0.277$ for a rock stratified in a natural manner in which the material property becomes weaker from bottom to surface. This says that the utilization of one-half of the surface velocity as an approximation to the actual base incident velocity could be as much as $80 \%$ in error, $(0.5-0.277) / 0.277$.

The initial pulse method considers only the initial wave behavior and doesn't include any influence from reflected waves. The method is independent of wave frequency, since frequency doesn't appear in the equation. Therefore, this method may not be totally correct in predicting the ratio, $r$. However, it does provide a first approximation to the prediction.

\section{FREQUENCY DOMAIN METHOD}

A more complete evaluation is possible by considering sine waves in the frequency domain. The equation that describes the vertical propagation of shearing waves without dissipation is

$$
\rho \frac{\partial^{2} u}{\partial t^{2}}=G \frac{\partial^{2} u}{\partial z^{2}}
$$

in which $z=$ distance in the vertical direction, $t=$ time, and $u=$ lateral displacement. For a sinusoidal wave the solution is,

$$
u(z, t)=U(z) \mathrm{e}^{i \omega t}
$$

in which

$$
U(z)=E \mathrm{e}^{i k z}+F \mathrm{e}^{-i k z}
$$

with

$$
k^{2}=\rho \omega^{2} / G
$$

$\omega=$ wave frequency in $\mathrm{rad} / \mathrm{sec}$

The time derivative of Eq. (10) gives the velocity, $V(z, t)$,

$$
V(z, t)=i \omega\left(E \mathrm{e}^{i k z}+F \mathrm{e}^{-i k z}\right) \mathrm{e}^{i \omega t}
$$

and the shear stress, $\tau(z, t)=G \mathrm{~d} u / \mathrm{d} z$, is given by

$$
\tau(z, t)=i k G\left(E \mathrm{e}^{i k z}-F \mathrm{e}^{-i k z}\right) \mathrm{e}^{i \omega t}
$$

In Eq. (13), the term $i \omega F \mathrm{e}^{i(k z+\omega t)}$ represents the incident wave traveling in the negative $z$-direction (upwards) and the term $i \omega F \mathrm{e}^{-i(k z-\omega t)}$ represents the reflected wave travelling in the positive $z$-direction (downwards) ${ }^{1,3}$. Corresponding incident and reflected shear waves may be identified in Eq. (14). 
Base excitation in one-dimensional soil dynamics: E. B. Wylie and C.-T. Wu

The total velocity and total shear stress at the top of a layer with thickness $h_{m}$, are

$$
\begin{aligned}
& V_{m}(0, t)=V_{U_{m}} \mathrm{e}^{i \omega t} \\
& \tau_{m}(0, t)=\tau_{U_{m}} \mathrm{e}^{i \omega t}
\end{aligned}
$$

in which

$$
\begin{aligned}
& V_{U_{m}}=i \omega\left(E_{m}+F_{m}\right) \\
& \tau_{U_{m}}=i k_{m} G_{m}\left(E_{m}-F_{m}\right)
\end{aligned}
$$

The constants for the layer may be expressed in terms of the given velocity and shear stress:

$$
\begin{aligned}
& E_{m}=-\frac{1}{2} i\left(\frac{V_{U_{m}}}{\omega}+\frac{\tau_{U_{m}}}{k_{m} \mathrm{G}_{m}}\right) \\
& F_{m}=-\frac{1}{2} i\left(\frac{V_{U_{m}}}{\omega}-\frac{\tau_{U_{m}}}{k_{m} \mathrm{G}_{m}}\right)
\end{aligned}
$$

The total velocity and total shear stress at the bottom of a layer with thickness $h_{m}$, are

$$
\begin{aligned}
& V_{m}\left(h_{m}, t\right)=V_{D_{m}} \mathrm{e}^{i \omega t} \\
& \tau_{m}\left(h_{m}, t\right)=\tau_{D_{m}} \mathrm{e}^{i \omega t}
\end{aligned}
$$

in which

$$
\begin{aligned}
& V_{D_{m}}=i \omega\left(E_{m} \mathrm{e}^{i k_{m} h_{m}}+F_{m} \mathrm{e}^{-i k_{m} h_{m}}\right) \\
& \tau_{D_{m}}=i k_{m} G_{m}\left(E_{m} \mathrm{e}^{i k_{m} h_{m}}-F_{m} \mathrm{e}^{-i k_{m} h_{m}}\right)
\end{aligned}
$$

From Eqs. (23) and (24):

$$
\begin{aligned}
& E_{m}=\frac{1}{2} i\left(\frac{V_{D_{m}}}{\omega}+\frac{\tau_{D_{m}}}{k_{m} G_{m}}\right) e^{-i k_{m} h_{m}} \\
& F_{m}=-\frac{1}{2} i\left(\frac{V_{D_{m}}}{\omega}-\frac{\tau_{D_{m}}}{K_{m} G_{m}}\right) e^{i k_{m} h_{m}}
\end{aligned}
$$

Equations (23) and (24) together with (17) and (18) yield

$$
\begin{gathered}
V_{D_{m}}=\cos k_{m} h_{m} \cdot V_{U_{m}}+i Z_{m} \sin k_{m} h_{m} \cdot \tau_{U_{m}} \\
\tau_{D_{m}}=\frac{\sin k_{m} h_{m}}{Z_{m}} \cdot V_{U_{m}}+\cos k_{m} h_{m} \cdot \tau_{U_{m}}
\end{gathered}
$$

in which

$$
Z_{m}=\omega / k_{m} G_{m}
$$

In Eqs. (15)-(29), $V_{U_{m}}, \tau_{U_{m}}, V_{D_{m}}$, and $\tau_{D_{m}}$ are functions of depth only. Eqs. (27) and (28) can be presented in matrix form

$$
\{\mathbf{D}\}_{m}=[\mathbf{F}]_{m}\{\mathbf{U}\}_{m}
$$

in which

$$
\begin{aligned}
& {[\mathbf{F}]_{m}=\left[\begin{array}{cc}
\cos k_{m} h_{m} & i Z_{m} \sin k_{m} h_{m} \\
\frac{i \sin k_{m} h_{m}}{Z_{m}} & \cos k_{m} h_{m}
\end{array}\right]} \\
& \{\mathbf{D}\}_{m}=\left\{\begin{array}{l}
V_{D_{m}} \\
\tau_{D_{m}}
\end{array}\right\} \\
& \{\mathbf{U}\}_{m}=\left\{\begin{array}{l}
V_{U_{m}} \\
\tau_{U_{m}}
\end{array}\right\}
\end{aligned}
$$

The total velocity and total shear stress at the bottom of the $(m-1)$ th layer is the total velocity and total shear stress at the top of the $m$ th layer,

$$
\begin{aligned}
& V_{m-1}\left(h_{m-1}, t\right)=V_{m}(0, t) \\
& \tau_{m-1}\left(h_{m-1}, t\right)=\tau_{m}(0, t)
\end{aligned}
$$

from which

$$
\begin{aligned}
& V_{D_{m-1}}=V_{U_{m}} \\
& \tau_{D_{m-1}}=\tau_{U_{m}}
\end{aligned}
$$

When the computation proceeds from layer $n$ upwards to layer 1, repeated application of Eqs. (30), (36) and (37) with index notation, $m$, changing from $n$ to 1 yields

$$
\{\mathbf{D}\}_{n}=[\mathbf{F}]_{1}[\mathbf{F}]_{2}[\mathbf{F}]_{3} \ldots[\mathbf{F}]_{n-1}[\mathbf{F}]_{n}\{\mathbf{U}\}_{1}
$$

Since the objective is to find the ratio, $r$, it is convenient to set the total surface velocity as a sine wave with unit amplitude and frequency $\omega$ :

$$
V_{1}(0, t)=\sin \omega t=\operatorname{Re}\left(-i \mathrm{e}^{i \omega t}\right)
$$

Eq. (36) together with $\tau_{1}=0$ defines the vector $\{\mathbf{U}\}_{1}$ as

$$
\{\mathbf{U}\}_{1}=\left\{\begin{array}{l}
-i \\
0
\end{array}\right\}
$$

and with the given $\rho_{m}, \mathrm{G}_{m}, \omega, h_{m}$, the transfer matrix $[\mathbf{F}]_{m}$ is defined by Eq. (31), where $m=1,2,3 \ldots, n$. By defining $[\mathbf{J}]$ as the product of the matrices $[\mathbf{F}]_{1},[\mathbf{F}]_{2} \ldots,[\mathbf{F}]_{n-1}$, $[\mathbf{F}]_{n}$,

$$
[\mathbf{J}]=\left[\begin{array}{ll}
J_{11} & J_{12} \\
J_{21} & J_{22}
\end{array}\right]
$$

in which $J_{11}, J_{12}, J_{21}$, and $J_{22}$ are independent of time, then Eq. (41) together with Eq. (38) gives

$$
\{\mathbf{D}\}_{n}=\left\{\begin{array}{l}
V_{D_{n}} \\
\tau_{D_{n}}
\end{array}\right\}=[\mathbf{J}]\{\mathbf{U}\}_{1}=\left[\begin{array}{ll}
J_{11} & J_{12} \\
J_{21} & J_{22}
\end{array}\right]\left\{\begin{array}{c}
-i \\
0
\end{array}\right\}
$$

Thus the total velocity and shear stress are available at level $n$ :

$$
\begin{aligned}
& V_{D_{n}}=-i J_{11} \\
& \tau_{D_{n}}=-i J_{21}
\end{aligned}
$$

Equations (43) and (44) together with (25) and (26) yield

$$
\begin{aligned}
& E_{n}=\frac{1}{2}\left(\frac{J_{11}}{\omega}+\frac{J_{21}}{k_{n} \mathrm{G}_{n}}\right) \mathrm{e}^{-i k_{n} h_{n}} \\
& F_{n}=-\frac{1}{2}\left(\frac{J_{11}}{\omega}-\frac{J_{21}}{k_{n} \mathrm{G}_{n}}\right) \mathrm{e}^{i k_{n} h_{n}}
\end{aligned}
$$

Once $E_{n}$ is defined, the amplitude of the incident velocity at the $n$th layer is defined as the modulus of $\omega E_{n}$ :

$$
\left|V_{I_{n}}\right|=\left|\omega E_{n}\right|
$$

Since the amplitude of the total surface velocity is unity in Eq. (39), one can obtain that the ratio between the amplitude of the base incident velocity and the amplitude of the total surface velocity is

$$
r=\left|\omega E_{n}\right|
$$

In Eq. (48), $\omega$ and $E_{n}$ are independent of time; thus, the ratio, $r$, obtained by this method is also independent of time. This method provides the layered system response in the frequency domain instead of the time domain. Therefore, it allows one to investigate the variation of $r$ with frequency and to find the critical frequencies at which $r$ is maximum or minimum.

Based on this method, several numerical examples are 


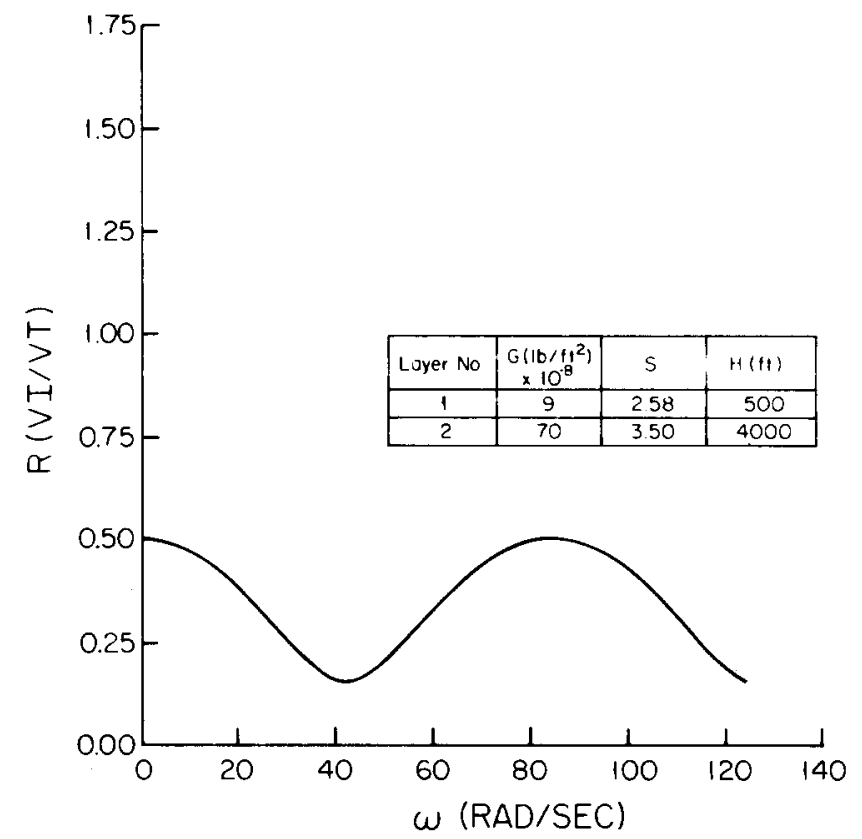

Figure 3. Velocity ratio vs. frequency, $n=1, T P 1$ stratification

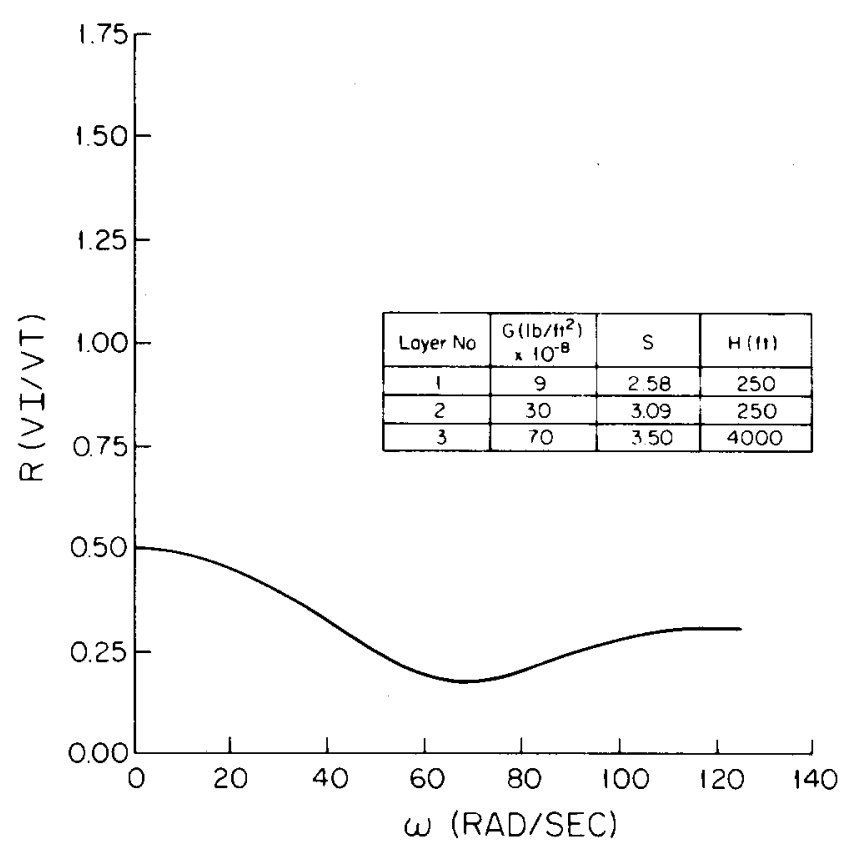

Figure 4. Velocity ratio vs. frequency, $n=2, T P I$ stratification

tested and illustrated. The type of stratification is TP1 (progressively weaker when measured from base to surface) for Figures 3-5, and TP2 (progressively stronger when measured from base to surface) for Figures 6-8. The wave frequency is varied in the range $1-125 \mathrm{rad} / \mathrm{sec}$, which includes the common frequencies of a seismic event. The number of layers above base level is one in Figures 3 and 6 , two in Figures 4 and 7, and nine in Figures 5 and 8. Figures 3-8 show how the velocity ratio, $r$, varies with the wave frequency for the specified rock in each case. It is useful to define the maximum and the minimum value of $r$ in Figures 3-5 as $r_{\max _{1}}$ and $r_{\min }$, and in Figures 6-8 as $r_{\text {max }_{2}}$ and $r_{\min }$. These limiting values represent the upper and the lower limits. Table 1 summarizes the findings.

When $r$ is plotted in the frequency domain, the result is a curve as shown in Figures 3-8. If the limiting values of $r$ are plotted in the frequency domain, the result becomes 2 points for each figure. This allows one to isolate these points from the frequency domain to see their relation with another dependent variable. These limiting values are plotted in Figure 9 to see their variation with the depth above base level $(H)$. A single symbol appears in Figure 9, to represent each case of a number of layers tested, i.e., six symbols represent 6 different numbers of layers, $n=1,2,3$, $5,9,30$. The figure shows that a critical depth exists beyond which no matter how the depth increases the values $r_{\min }$ and $r_{\max }$ remain constant in this frequency range. This critical depth is important in identifying potential errors in the use of $r=0.5$. Table 2 shows the critical values, identified as $H c_{1}$ for $r_{\text {min }_{1}}$ and $H c_{2}$ for $r_{\max _{2}}$.

The use of one-half surface velocity $(r=0.5)$ to replace the base incident velocity will be either an overestimation

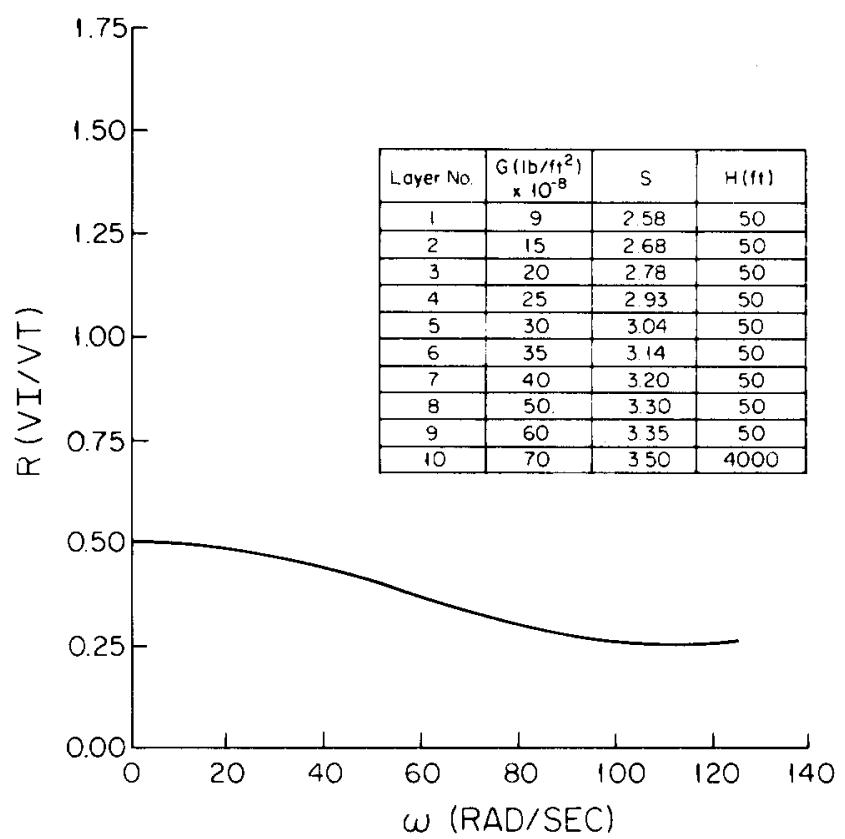

Figure 5. Velocity ratio vs. frequency, $n=9, T P 1$ stratification

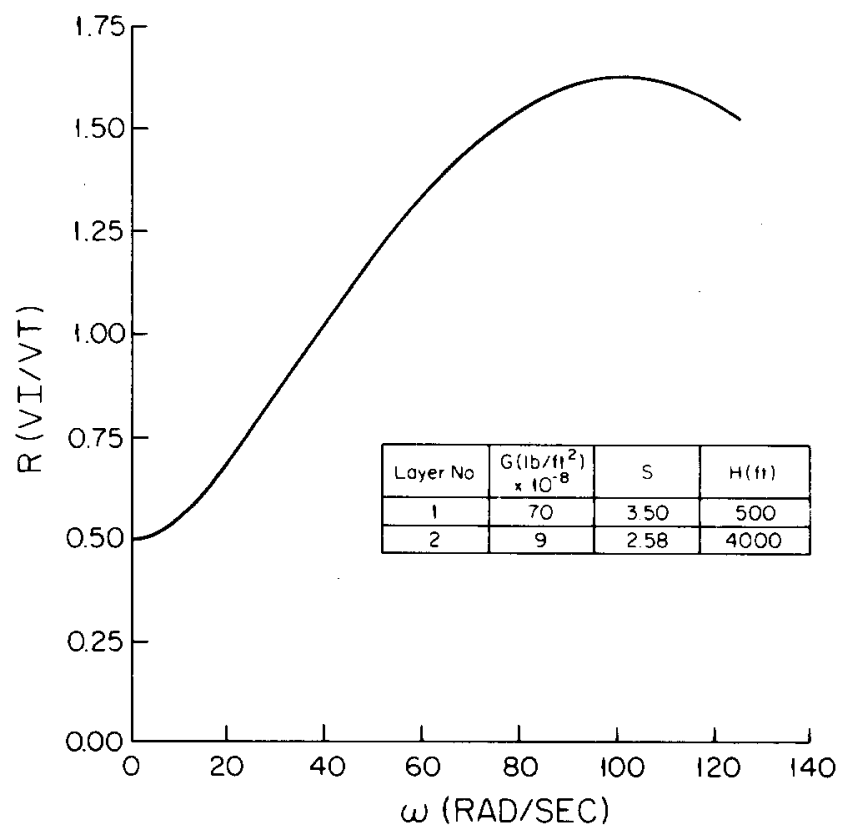

Figure 6. Velocity ratio vs. frequency, $n=1, T P 2$ stratification 
Base excitation in one-dimensional soil dynamics: E. B. Wylie and C.-T. Wu

for TP1 rock or an underestimation for the TP2 rock. If the possible maximum errors resulting from this approximation are represented by $P_{1}$ and $P_{2}$ for TP1 and TP , respectively, then,

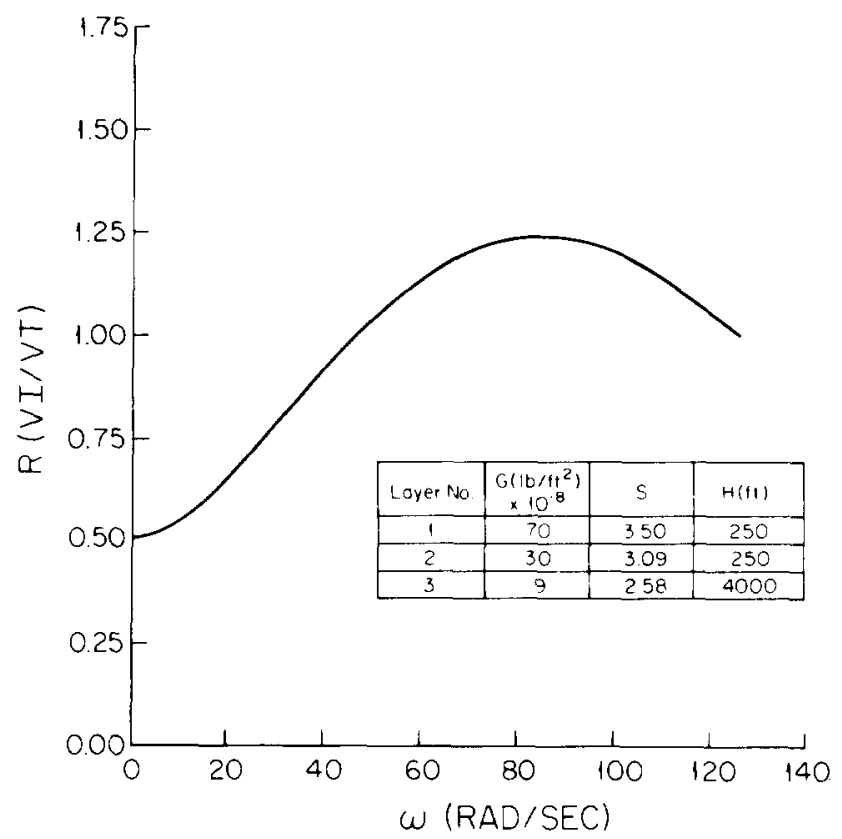

Figure 7. Velocity ratio vs. frequency, $n=2, T P 2$ stratification

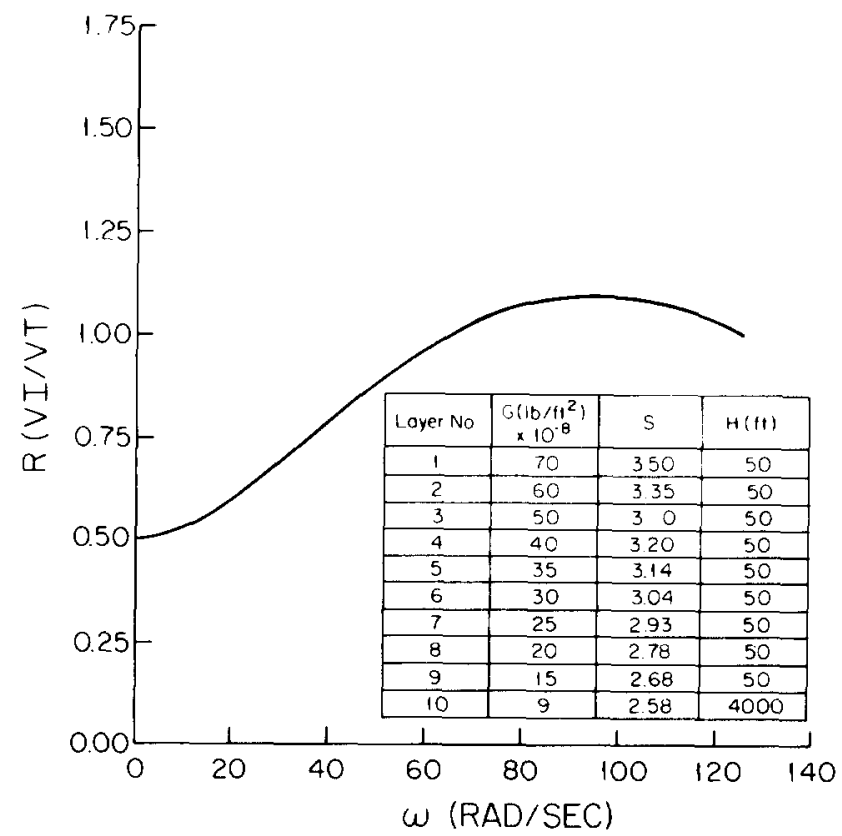

Figure 8. Velocity ratio vs. frequency, $n=9, T P 2$ stratification

$$
\begin{aligned}
& P_{1}=\left(0.5-r_{\text {min }_{1}}^{\prime}\right) / r_{\text {min }_{1}}^{\prime} \\
& P_{2}=\left(0.5-r_{\text {max }_{2}}^{\prime}\right) / r_{\text {max }_{2}}^{\prime}
\end{aligned}
$$

$r_{\min _{1}}^{\prime}=$ smallest $r_{\min _{1}}$ in Figure 9, $r_{\max _{2}}^{\prime}=$ largest $r_{\max _{2}}$ in Figure 9. Table 2 summarizes the results from Figure 9. In addition to the specific findings in Table 2, the following two general comments apply.

\begin{tabular}{|c|c|c|c|c|c|}
\hline No. & $\begin{array}{c}\text { Figure } \\
\text { no. }\end{array}$ & $\begin{array}{c}\text { Type of } \\
\text { stratification }\end{array}$ & $n$ & $\begin{array}{l}\text { critical freque } \\
\text { amplitude }\end{array}$ & ency \\
\hline 1 & 3 & TP1 & 1 & $\begin{array}{l}\omega=1 \mathrm{rad} / \mathrm{sec} \\
\omega=41 \mathrm{rad} / \mathrm{sec}\end{array}$ & $\begin{array}{l}r_{\text {max }_{1}}=0.5 \\
r_{\text {min }_{1}}=0.15\end{array}$ \\
\hline 2 & 4 & TP1 & 2 & $\begin{array}{l}\omega=1 \mathrm{rad} / \mathrm{sec} \\
\omega=68 \mathrm{rad} / \mathrm{sec}\end{array}$ & $\begin{array}{l}r_{\max _{1}}=0.5 \\
r_{\text {min }_{1}}=0.18\end{array}$ \\
\hline 3 & - & TP1 & 5 & $\begin{array}{l}\omega=1 \mathrm{rad} / \mathrm{sec} \\
\omega=125 \mathrm{rad} / \mathrm{sec}\end{array}$ & $\begin{array}{l}r_{\max _{1}}=0.5 \\
r_{\min _{1}}=0.25\end{array}$ \\
\hline 4 & 5 & TP1 & 9 & $\begin{array}{l}\omega=1 \mathrm{rad} / \mathrm{sec} \\
\omega=110 \mathrm{rad} / \mathrm{sec}\end{array}$ & $\begin{array}{l}r_{\max _{1}}=0.5 \\
r_{\text {min }_{1}}=0.26\end{array}$ \\
\hline 5 & 6 & TP2 & 1 & $\begin{array}{l}\omega=98 \mathrm{rad} / \mathrm{sec} \\
\omega=1 \mathrm{rad} / \mathrm{sec}\end{array}$ & $\begin{array}{l}r_{\max _{2}}=1.64 \\
r_{\min _{2}}=0.5\end{array}$ \\
\hline 6 & 7 & TP2 & 2 & $\begin{array}{l}\omega=86 \mathrm{rad} / \mathrm{sec} \\
\omega=1 \mathrm{rad} / \mathrm{sec}\end{array}$ & $\begin{array}{l}r_{\max _{2}}=1.25 \\
r_{\min _{2}}=0.5\end{array}$ \\
\hline 7 & - & TP2 & 5 & $\begin{array}{l}\omega=104 \mathrm{rad} / \mathrm{sec} \\
\omega=1 \mathrm{rad} / \mathrm{sec}\end{array}$ & $\begin{array}{l}r_{\text {max }_{2}}=1.15 \\
r_{\min _{2}}=0.5\end{array}$ \\
\hline 8 & 8 & TP2 & 9 & $\begin{array}{l}\omega=94 \mathrm{rad} / \mathrm{sec} \\
\omega=1 \mathrm{rad} / \mathrm{sec}\end{array}$ & $\begin{array}{l}r_{\max _{2}}=1.09 \\
r_{\min _{2}}=0.5\end{array}$ \\
\hline
\end{tabular}

Table 1. Sample value of velocity ratio $r$ in different rock stratifications

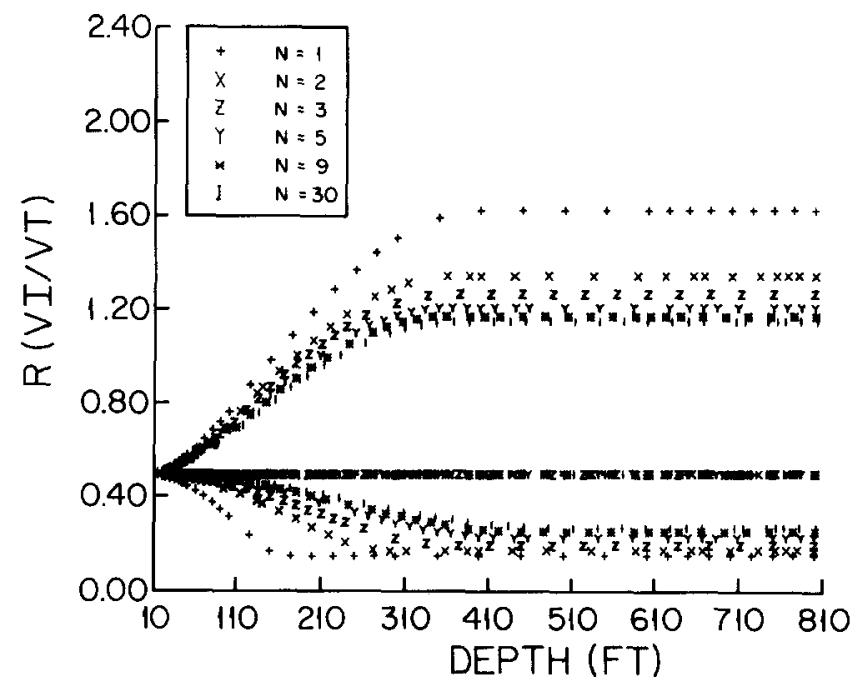

Figure 9. Extreme values of velocity ratio vs. total depth, $H$

\begin{tabular}{|c|c|c|c|c|c|c|c|c|}
\hline$n$ & $H c_{1}$ & $\mathrm{Hc}_{2}$ & $H>H c_{1}$ & $H<H c_{1}$ & $H>H c_{2}$ & $H<H c_{2}$ & $P_{1}$ & $P_{2}$ \\
\hline 1 & $160^{\prime}$ & $400^{\prime}$ & $r_{\min _{1}}^{\prime}=0.15$ & $0.15<r_{\min _{1}}<0.5$ & $r_{\text {max }_{2}}^{\prime}=1.65$ & $0.5<r_{\max _{2}}<1.65$ & 2.33 & -0.70 \\
\hline 2 & $250^{\prime}$ & $360^{\prime}$ & $r_{\min _{1}}^{\prime}=0.19$ & $0.19<r_{\text {min }_{1}}<0.5$ & $r_{\max _{2}}^{\prime}=1.34$ & $0.5<r_{\text {max }_{2}}<1.34$ & 1.63 & -0.63 \\
\hline 3 & $330^{\prime}$ & $340^{\prime}$ & $r_{\min _{1}}^{\prime}=0.23$ & $0.23<r_{\text {min }_{1}}<0.5$ & $r_{\max _{2}}^{\prime}=1.27$ & $0.5<r_{\max _{2}}<1.27$ & 1.17 & -0.61 \\
\hline 5 & $380^{\prime}$ & $330^{\prime}$ & $r_{\min _{1}}^{\prime}=0.28$ & $0.28<r_{\text {min }_{1}}<0.5$ & $r_{\max _{2}}^{\prime}=1.20$ & $0.5<r_{\text {max }_{2}}<1,20$ & 0.78 & -0.58 \\
\hline 9 & $400^{\prime}$ & $320^{\prime}$ & $r_{\min _{1}}^{\prime}=0.30$ & $0.30<r_{\text {min }_{1}}<0.5$ & $r_{\max _{2}}^{\prime}=1.12$ & $0.5<r_{\max _{2}}<1.12$ & 0.67 & -0.55 \\
\hline 30 & $420^{\prime}$ & $315^{\prime}$ & $r_{\min _{1}}^{\prime}=0.34$ & $0.34<r_{\text {min }_{1}}<0.5$ & $r_{\max _{2}}^{\prime}=1.07$ & $0.5<r_{\max _{2}}<1.07$ & 0.47 & -0.53 \\
\hline
\end{tabular}

Table 2. Maximum and minimum values of velocity ratio, Figure 9 
(1) the usage of one-half of the surface velocity as the incident velocity at the base level would generate an excessively large soil response (conservative) for TP1 rock. This is so since, in every case, less than $50 \%$ of the surface velocity is actually needed but $50 \%$ would be used.

(2) the usage of one-half the surface velocity as the incident velocity in TP2 rock underestimates the soil behaviour (nonconservative), since more than $50 \%$ surface velocity is actually needed but $50 \%$ would be used.

The change of rigidity and mass density between two layers are given as

$$
\begin{aligned}
\Delta \mathrm{G} & =\frac{6.1 \times 10^{9}}{n} \\
\Delta \rho & =\frac{1.79}{n}
\end{aligned}
$$

Equations (51) and (52) show that $\Delta \mathrm{G}$ and $\Delta \rho$ vary inversely with $n$. Thus in the previous example, $\Delta \mathrm{G}_{1}>\Delta \mathrm{G}_{2}>\Delta \mathrm{G}_{3}>\Delta \mathrm{G}_{5}>\Delta \mathrm{G}_{9}>\Delta \mathrm{G}_{30}$, and $\Delta \rho_{1}>\Delta \rho_{2}>\Delta \rho_{3}>\Delta \rho_{5}>\Delta \rho_{9}>\Delta \rho_{30}$. Numerical subscripts refer to the layer number. An interesting finding from this is that $P_{1}$ and $\left|P_{2}\right|$ increase with $\Delta \mathrm{G}$ and $\Delta \rho$. The case $(n=30, T P 1)$ is a simulation of an ordinary rock in which the rigidity and mass density decrease towards the free surface.

\section{INFLUENCE OF VISCOSITY ON THE PREDICTION}

Base material without dissipation was discussed in the previous illustration. Now it is desired to take account of the effect of viscosity. When the viscosity, $\mu$, is considered in the computation of the velocity equation in the frequency domain, the shear modulus is modified from $G_{n}$ to $G_{n}^{*}$.

$$
G_{n}^{*}=G_{n}+i \omega \mu
$$

The critical damping ratio ${ }^{2}, \beta$, is related to viscosity, $\mu$, shear modulus, $G$, and frequency, $\omega$, by

$$
\beta=\frac{\omega \mu}{2 G}
$$

Rock generally can be described with a viscosity of less than $3000 \mathrm{lb} \mathrm{sec} / \mathrm{ft}^{2}$. Thus the maximum damping ratio $\beta$ is less than $0.03 \%$. Since the maximum damping ratio is small, one may expect the influence of viscosity also to be small. In order to check this, the same cases as Figure 9, but with viscosity $3000 \mathrm{lb} \mathrm{sec} / \mathrm{ft}^{2}$, were computed. The results, when compared with Figure 9, show that the inclusion of viscosity essentially produced identical curves. It is noted that energy dissipation and viscous effects play an important role in soil behaviour during seismic events. However the objective here is to find the velocity ratio, $r$, in rock to be utilized as excitation at the soil base; therefore, only the rock viscosity needs to be considered.

\section{CONCLUSIONS}

1. The velocity ratio, $r$, which determines the percentage of rock surface velocity that should be used as the excitation at the transmitting boundary at a soil base, is in the range $0.15-1.65$.
2. If field data shows that the rock property is of the type that becomes weaker toward the surface, the usage of onehalf surface velocity is proper in the engineering sense, since the approximation is conservative.

3. If field data shows that the rock property is opposite, the usage of $165 \%$ of the rock surface velocity would provide a conservative computation. It is noted that rock of this type is very unusual and probably seldom exists.

4. The multilayered case ( $n=30, T P 1)$ is probably the most practical one since it is a simulation of an ordinary rock whose property (shear modulus, mass density, etc.), becomes weaker toward the surface. If the rock actually consists of multilayers (e.g., $n=100,1000, \ldots)$, or displays a continuous variation in properties, the range of $r$ will become narrower and closer to 0.5 . Therefore, it may be a good approximation to use one-half of the rock surface velocity as the base incident velocity.

5 . The ratio, $r$, is obtained from a sinusoidal velocity with frequency, $\omega$, but can be extended to a seismic motion with dominant frequency, $\omega_{0}$. It requires first to discretize the base period, $T_{0}$, and to expand the surface rock velocity into Fourier series, then the remaining procedure is similar to the frequency domain method ${ }^{4,7}$.

6. The first pulse method, although a gross approximation, provides a simple equation that shows that the velocity ratio, $r$, is less than 0.5 for TP1 rock and greater than 0.5 for TP2 rock. The variation of $r$ is similar to the results from the frequency domain method, i.e., $0.28<r<1.65$. For a large number of layers, $n \geqslant 30$, the two predictions agree quite well.

\section{ACKNOWLEDGEMENT}

The investigation described herein was supported by National Science Foundation Grant No. PFR-8017429 to The University of Michigan. The assistance of Professor F. E. Richart, Jr., who made helpful suggestions during the preparation of the manuscript, is also acknowledged.

\section{REFERENCES}

1. Cooley, J. W. and Tukey, J. W., "An Algorithm for the Machine Calculation of Complex Fourier Series", Mathematics of Computation, Vol. 19, No. 90, 1965, pp. 297-301.

2. Hardin, B. D. and Drnevich, V. P. "Shear Modulus and Damping in Soils: Measurement and Parameter Effects", Journal of the Soil Mechanics and Foundations Division, ASCE, Vol. 98, No. SM6. Proc. Paper 8977, pp. 603-624.

3. Joyner, W. B. and Chen, A. F. T. "Calculation of Nonlinear Ground Response in Earthquakes", Bulletin of the Seismological Society of America, Vol. 65, No. 5, Oct. 1975, pp. 1315-1336.

4. Schnabel, P. B., Lysmer, J. and Seed, H. B. "SHAKE: A Computer Program for Earthquake Response Analysis of Horizontally Layered Sites", A report on Research Sponsored by the National Science Foundation, Report No. EERC 72-12, Dec., 1972.

5. Wuerker, R. G."Annotated Tables of Strength and Elastic Properties of Rocks", University of Illinois, Department of Mining and Metallurgical Engineering, Published by Petroleum Branch, AIME, Dec., 1956

6. Streeter, V. L., Wylie, E. B. and Richart, F. E. "Soil Motion Computations by Characteristic Method", Journal of the Geotechnical Engineering Division, ASCE, Vol. 100, No. GT3, March 1974

7. Wylie, E. B. and Streeter, V. L. "Fluid Transients", McGraw-Hill, 1978, pp. 231-232

APPENDIX. Limiting value of velocity ratio.

$$
\text { Let } \prod_{m=2}^{n} A_{m-1}=\left(\frac{\rho_{1} \mathrm{G}_{1}}{\rho_{n} \mathrm{G}_{n}}\right)^{1 / 2}=C
$$


The purpose is to prove that $r$ in Eq. (7) has a minimum value $(\neq 0)$ when $A_{1}=A_{2}=A_{3}=\ldots=A_{n-1}=C^{1 /(n-1)}$ even if $n$ approaches infinity. TP1 rock is discussed but the same procedure could be applied to TP2 rock to prove that TP2 rock has a maximum value $(\neq \infty)$. Let

$$
l_{m-1}=\frac{\rho_{m-1} \mathrm{G}_{m-1}}{\rho_{m} \mathrm{G}_{m}}
$$

then,

$$
r=\frac{1}{2}\left(\prod_{m-2}^{n} \frac{1+l_{m}^{1 / 2}-1}{2}\right)
$$

Applying Taylor series expansion about $l_{m-1}=1$ and assuming $\left(l_{m-1}-1\right)$ is small enough so that the higher order terms can be neglected:

$$
l_{m-1}^{1 / 2} \approx 1+1 / 2\left(l_{m-1}-1\right)
$$

so

$$
r=1 / 2\left(\prod_{m=2}^{n} \frac{2+1 / 2\left(l_{m-1}-1\right)}{2}\right)
$$

Since $\left(l_{m-1}-1\right)$ is assumed very small, Eq. (59) can be approximated as

$$
\begin{aligned}
r & =\frac{1}{2^{n}}\left[2^{n-1}+2^{n-2} 1 / 2 \sum_{m=2}^{n}\left(l_{m-1}-1\right)\right] \\
& =1 / 2+1 / 8\left[\left(\sum_{m=2}^{n} l_{m-1}\right)-(n-1)\right]
\end{aligned}
$$

Applying the Cauchy inequality equation,

$$
\frac{1}{n-1}\left(\sum_{m=2}^{n} l_{m-1}\right) \geqslant\left(\prod_{m=2}^{n} l_{m-1}\right)^{\frac{1}{n-1}}
$$

Eq. (61) says that $\sum_{m=2}^{n} l_{m-1}$ has a minimum value when $l_{1}=l_{2}=l_{3}=\ldots=l_{n-1}^{m=2}$ or $A_{1}=A_{2}=A_{3}=\ldots=A_{n-1}$; thus

$$
A_{1}=A_{2}=A_{3}=\ldots=A_{n-1}=C^{1 /(n-1)}
$$

TP1 rock has a minimum $r$ when $S_{1}=2.58$ and $\mathrm{G}_{1}=9 \times 10^{8} \mathrm{lb} / \mathrm{ft}^{2}$, and $S_{n}=3.50$ and $\mathrm{G}_{n}=7 \times 10^{9} \mathrm{lb} / \mathrm{ft}^{2}$. Therefore, $C$ is 0.3077 , and

$$
r_{\min _{1}}=1 / 2\left(\prod_{m=2}^{n} \frac{1+C^{1 /(n-1)}}{2}\right)
$$

By taking $n=2,5,10,100,1000$ and 10000 , the following values of $r_{\min _{1}}$ are computed

$$
\begin{array}{r}
r_{\text {min }_{1}}(n=2)=0.3269 \\
r_{\text {min }_{1}}(n=5)=0.2896 \\
r_{\text {min }_{1}}(n=10)=0.2828 \\
r_{\text {min }_{1}}(n=100)=0.2778 \\
r_{\text {min }_{1}}(n=1000)=0.2774 \\
r_{\text {min }_{1}}(n=10000)=0.2772
\end{array}
$$

From these values one can see that $r_{\min _{1}}$ approaches 0.277 as $n$ approaches infinity. It is noted that when $n=100$, $l_{m-1}=0.9765$, and when $n=1000, l_{m-1}=0.9976$; therefore, $\left(l_{m-1}-1\right)$ is small when $n$ is large. 